\title{
Nonlinear extensions of Schrödinger-von Neumann quantum dynamics: a list of conditions for compatibility with thermodynamics
}

\author{
Gian Paolo Beretta* \\ Istituto Nazionale di Fisica della Materia, Università di Brescia, via Branze 38, 25123 Brescia, Italy
}

(Dated: October 11, 2018)

\begin{abstract}
We propose a list of conditions that consistency with thermodynamics imposes on linear and nonlinear generalizations of standard unitary quantum mechanics that assume a set of true quantum states without the restriction $\rho^{2}=\rho$ even for strictly isolated systems and that are to be considered in experimental tests of the existence of intrinsic (spontaneous) decoherence at the microscopic level.
\end{abstract}

PACS numbers: 03.65.Ta,03.65.Yz,03.67.-a,05.70.-a

Understanding and predicting 'decoherence' is important in future applications involving nanometric devices, fast switching times, clock synchronization, superdense coding, quantum computation, teleportation, quantum cryptography, etc. where entanglement structure and dynamics play a key role [1]. In the last three decades it has also been central in exploring possible limitations to the validity of standard unitary quantum mechanics (QM), by studying a variety of linear and nonlinear extensions that have been advocated by several authors on a variety of conceptual grounds [2]. It has been suggested 3] that long-baseline neutrino oscillation experiments may provide means of testing the existence of spontaneous decoherence at the microscopic level and the validity of linear and nonlinear extensions of the Schrödingervon Neumann equation of motion of QM, thus prompting a renewed interest on such extensions $3,[4,5]$.

The aim of this Letter is to list the main conditions that must be imposed and checked on linear and nonlinear extensions of QM which assume an augmented set of true quantum states described by state operators $\rho$ without the restriction $\rho^{2}=\rho$. The reasoning and framework proposed here should provide useful guidance also to current efforts to define general measures of entanglement [6].

The conditions proposed here form a very restrictive set. Yet, at least one possible extension has been proved to satisfy them all [7], with mathematics that has been partially rediscovered recently by researchers in different contexts and fields [5, 8, 9].

\section{Causality. Forward and backward in time.}

We consider the set $\mathcal{P}$ of all linear, hermitian, nonnegative-definite, unit-trace operators $\rho$ (without the restriction $\rho^{2}=\rho$ ) on the standard QM Hilbert space $\mathcal{H}$ associated with a strictly isolated system 10. Every solution of the equation of motion, i.e., every trajectory $u(t, \rho)$ passing at time $t=0$ through state $\rho$ in $\mathcal{P}$, should lie entirely in $\mathcal{P}$ for all times $t,-\infty<t<+\infty$. This strong causality condition is nontrivial and demanding both from the conceptual and the technical mathematical points of view.

2. Conservation of energy and other invariants.
The value of the energy functional $e(\rho)=\operatorname{Tr}(\rho H)$, where $H$ is the standard QM Hamiltonian operator associated with the isolated system $[H \neq H(t)]$, must remain invariant along every trajectory. If $\mathcal{H}$ is the Fock space of an isolated system consisting of $M$ types of elementary constituents (e.g., atoms and molecules if chemical and nuclear reactions are inhibited; or atomic nuclei and electrons for modelling chemical reactions) each with a number operator $N_{i}\left(\left[H, N_{i}\right]=0\right.$ and $\left.\left[N_{i}, N_{j}\right]=0\right)$, then also the value of each number-of-constituents functional $n_{i}(\rho)=\operatorname{Tr}\left(\rho N_{i}\right)$ must remain invariant along every trajectory. Depending on the type of system, there may be other time-invariant functionals, e.g., the total momentum components $p_{j}(\rho)=\operatorname{Tr}\left(\rho P_{j}\right)$, with $j=x, y, z$, for a free particle (in which case Galileian invariance must also be verified, for $\left[H, P_{j}\right]=0$ and $\left.\left[P_{i}, P_{j}\right]=0\right)$. In what follows, we denote by $g_{i}(\rho)=\operatorname{Tr}\left(\rho G_{i}\right)$ the set of non-Hamiltonian time-invariant functionals, if any, with $\left[H, G_{i}\right]=0$ and $\left[G_{i}, G_{j}\right]=0$ (clearly, $H$ and the $G_{i}$ 's have a common eigenbasis that we denote by $\left\{\left|\psi_{\ell}\right\rangle\right\}$ ).

3. Standard QM unitary evolution of $\rho^{2}=\rho$ states. Unitary time evolution of the states of QM according to the Schrödinger equation of motion must be compatible with the more general dynamical law. These trajectories, passing through any state $\rho$ such that $\rho^{2}=\rho$ and entirely contained in the state domain of QM, must be solutions also of the extended dynamical law. Because the states of QM are extreme points of the state domain $\mathcal{P}$, the trajectories of QM must be boundary solutions (limit cycles) of the extended dynamical law.

In general, any extended dynamical equation may be written in the form

$$
\begin{aligned}
& \frac{\mathrm{d} \rho}{\mathrm{d} t}=-\frac{i}{\hbar}[H, \rho]+D_{M} \\
& \text { with } D_{M}=\hat{\mathbf{D}}_{M}\left(\rho, H, G_{i}, \ldots\right),
\end{aligned}
$$

where operator $D_{M}$ represents the dissipative part of the equation of motion and may depend linearly and/or nonlinearly (through superoperator $\hat{\mathbf{D}}_{M}$ ) on the state operator $\rho$, on the Hamiltonian $H$, on the linear operators $G_{i}$ associated with the other time invariants (if any), as well as on the structure and the number $M$ of elemen- 
tary constituents of the system. Like the Schrödingervon Neumann term, also the dissipative term should not be responsible for rates of change of any of the invariant functionals $\operatorname{Tr}(\rho), e(\rho), g_{i}(\rho)$ and, therefore,

$$
\operatorname{Tr} D_{M}=0 \quad \operatorname{Tr} D_{M} H=0 \quad \operatorname{Tr} D_{M} G_{i}=0 .
$$

If the complete dynamics preserves the feature of uniqueness of solutions throughout the state domain $\mathcal{P}$, then pure states can only evolve according to the Schrödinger equation of motion and, therefore, $\hat{\mathbf{D}}_{M}\left(\rho, H, G_{i}, \ldots\right)=0$ when $\rho^{2}=\rho$. This feature that may be responsible for hiding the presence of deviations from QM in experiments where the isolated system is prepared in a pure state. It also implies that no trajectory can enter or leave the state domain of QM. Thus, by continuity, there must be trajectories that approach indefinitely these boundary solutions (of course, this can only happen backward in time, as $t \rightarrow-\infty$, for otherwise the entropy of the isolated system would decrease in forward time).

\section{Conservation of effective Hilbert space dimen- sionality.}

Unitary dynamics [Eq. (11) with $D_{M}=0$ ] would maintain unchanged all the eigenvalues of $\rho$ and therefore cannot satisfy Condition 5 below [14]. Instead, we only require that the dynamical law maintains zero the initially zero eigenvalues of $\rho$ and, therefore, conserves the cardinality of the set of zero eigenvalues, $\operatorname{dim} \operatorname{Ker}(\rho)$. In other words, if the isolated system is prepared in a state that does not 'occupy' the eigenvector $\left|\psi_{\ell}\right\rangle$ of $H$ (and the $G_{i}$ 's), i.e., if $\rho(0)\left|\psi_{\ell}\right\rangle=0$ (so that $\left|\psi_{\ell}\right\rangle$ is also an eigenvector of $\rho$ corresponding to a zero eigenvalue), then such energy eigenvector remains 'unoccupied' at all times, i.e., $\rho(t)\left|\psi_{\ell}\right\rangle=0$.

This condition preserves an important feature that allows remarkable model simplifications within QM: the dynamics is fully equivalent to that of a model system with Hilbert space $\mathcal{H}^{\prime}$ (a subspace of $\mathcal{H}$ ) defined by the linear span of all the $\left|\psi_{\ell}\right\rangle$ 's such that $\rho(t)\left|\psi_{\ell}\right\rangle \neq 0$ at some time $t$ (and, hence, by our condition, at all times). The relevant operators $X^{\prime}$ on $\mathcal{H}^{\prime}\left(\rho^{\prime}, H^{\prime}, G_{i}^{\prime}, \ldots\right)$ are defined from the original $X$ on $\mathcal{H}\left(\rho, H, G_{i}, \ldots\right)$ so that $\left\langle\alpha_{k}\left|X^{\prime}\right| \alpha_{\ell}\right\rangle=\left\langle\alpha_{k}|X| \alpha_{\ell}\right\rangle$ with $\left|\alpha_{k}\right\rangle$ any basis of $\mathcal{H}^{\prime}$.

It is also consistent with recent experimental tests 11 that rule out, for pure states, deviations from linear and unitary dynamics and confirm that initially unoccupied eigenstates cannot spontaneously become occupied. This fact adds nontrivial experimental and conceptual difficulty to the problem of designing a fundamental test of QM, capable of ascertaining whether decoherence originates from uncontrolled interactions with the environment due to the practical impossibility of obtaining strict isolation, or else it is a more fundamental intrinsic feature of microscopic dynamics requiring an extension of QM. In the latter case, this condition will preserve within the extended theory the exact validity of all the remarkable successes of QM.

5. Entropy nondecrease. Irreversibility. The principle of nondecrease of entropy [16] for an isolated system must be satisfied, i.e., the rate of change of the entropy functional $-k_{\mathrm{B}} \operatorname{Tr}(\rho \ln \rho)$ must be nonnegative along every trajectory, $-k_{\mathrm{B}} \operatorname{Tr}[u(t, \rho) \ln u(t, \rho)] \geq-k_{\mathrm{B}} \operatorname{Tr}(\rho \ln \rho)$.

6. Stability and uniqueness of the thermodynamic equilibrium states. Second law. A state operator $\rho$ of the isolated system represents an equilibrium state if $\mathrm{d} \rho / \mathrm{d} t=0$. For each given set $(\tilde{e}, \tilde{\mathbf{g}})$ of feasible values of the energy functional $e(\rho)$ and the other time-invariant functionals $g_{i}(\rho)$, if any, among all the equilibrium states that the dynamical law may admit there must be one and only one which is globally stable 13 .

This stable equilibrium state must be that of equilibrium thermodynamics and, therefore, of the form

$$
\rho_{\mathrm{e}}=\frac{\exp \left[-\beta(\tilde{e}, \tilde{\mathbf{g}}) H+\sum_{i} \nu_{i}(\tilde{e}, \tilde{\mathbf{g}}) G_{i}\right]}{\operatorname{Tr} \exp \left[-\beta(\tilde{e}, \tilde{\mathbf{g}}) H+\sum_{i} \nu_{i}(\tilde{e}, \tilde{\mathbf{g}}) G_{i}\right]},
$$

where $G_{i}$ are defined above. Of course, states given by Eq. (41) are solutions of the constrained maximization problem

$$
\begin{aligned}
& \max -k_{\mathrm{B}} \operatorname{Tr}(\rho \ln \rho) \text { subject to } \\
& \operatorname{Tr}(\rho)=1, \operatorname{Tr}(\rho H)=\tilde{e}, \operatorname{Tr}\left(\rho G_{i}\right)=\tilde{g}_{i}, \text { and } \rho \geq(55)
\end{aligned}
$$

and reduce to the canonical equilibrium states $\rho_{\mathrm{e}}=$ $\exp (-\beta H) / \operatorname{Tr} \exp (-\beta H)$ when $G_{i}=g_{i} I$ for all $i$ 's (with $g_{i}$ scalars and $I$ the identity on $\left.\mathcal{H}\right)$, and to the microcanonical state $\rho_{\mathrm{e}}=I / \operatorname{dim} \mathcal{H}$ if also $H=e I$ (and $\operatorname{dim} \mathcal{H}<\infty)$.

As discussed in Ref. [15], the entropy functional is not a Lyapunov function, even if, in a strict sense that depends on the continuity and the conditional stability of states $\rho_{\mathrm{e}}$, it does provide a criterion for the local stability of these states. In addition to this, the second law requires however that no other equilibrium state of the dynamical law be be globally stable 13,14 .

Consider the noteworthy family of states

$$
\rho_{\mathrm{nd}}=\frac{B \exp \left[-\beta(\tilde{e}, \tilde{\mathbf{g}}) H+\sum_{i} \nu_{i}(\tilde{e}, \tilde{\mathbf{g}}) G_{i}\right] B}{\operatorname{Tr} B \exp \left[-\beta(\tilde{e}, \tilde{\mathbf{g}}) H+\sum_{i} \nu_{i}(\tilde{e}, \tilde{\mathbf{g}}) G_{i}\right]},
$$

where $B$ is any given idempotent operator $B^{2}=B$. This family, which includes pure states $[\operatorname{Tr}(B)=1]$, maximizes the entropy [Prob. (5)] subject to the additional constraint $\rho=B \rho B$ for the given $B$. All eigenvalues of $\rho_{\text {nd }}$ must remain invariant (otherwise the entropy would decrease) and the state is equilibrium if $[B, H]=0$ or otherwise it evolves unitarily (limit cycle) with $B(t)=\exp (-i H t / \hbar) B(0) \exp (i H t / \hbar)$. They have a thermal-like distribution (positive and negative temperatures) over a finite number $[\operatorname{Tr}(B)]$ of 'occupied' eigenvectors. Because entropy cannot decrease and $-k_{\mathrm{B}} \operatorname{Tr}(\rho \ln \rho)$ is an $S$-function, they are conditionally locally stable 
equilibrium states or limit cycles [15]. For them not to be globally stable, as required by the second law, it suffices that the extended dynamics imply that at least one state of equal energy and other invariants (not necessarily neighboring nor with the same kernel) evolves towards higher entropy than $\rho_{\text {nd }}$.

7. Non-interacting subsystems. Separate energy conservation. For an isolated system composed of two distinguishable subsystems $A$ and $B$ with associated Hilbert spaces $\mathcal{H}^{A}$ and $\mathcal{H}^{B}$, so that the Hilbert space of the system is $\mathcal{H}=\mathcal{H}^{A} \otimes \mathcal{H}^{B}$, if the two subsystems are non-interacting, i.e., the Hamiltonian operator $H=$ $H_{A} \otimes I_{B}+I_{A} \otimes H_{B}$, then the functionals $\operatorname{Tr}\left[\left(H_{A} \otimes I_{B}\right) \rho\right]=$ $\operatorname{Tr}_{A}\left(H_{A} \rho_{A}\right)$ and $\operatorname{Tr}\left[\left(I_{A} \otimes H_{B}\right) \rho\right]=\operatorname{Tr}_{B}\left(H_{B} \rho_{B}\right)$ represent the energies of the two subsystems and must remain invariant along every trajectory, even if the states of $A$ and $B$ are correlated, i.e., even if $\rho \neq \rho_{A} \otimes \rho_{B}$. Of course, $\rho_{A}=\operatorname{Tr}_{B}(\rho), \rho_{B}=\operatorname{Tr}_{A}(\rho), \operatorname{Tr}_{B}$ denotes the partial trace over $\mathcal{H}^{B}$ and $\operatorname{Tr}_{A}$ the partial trace over $\mathcal{H}^{A}$.

8. Independent states. Weak separability. Separate entropy nondecrease. Two distinguishable subsystems $A$ and $B$ are in independent states if the state operator $\rho=\rho_{A} \otimes \rho_{B}$. For any given $\rho$, let us define the idempotent operator $B$ obtained from $\rho$ by substituting in its spectral expansion each nonzero eigenvalue with unity [12] and the entropy operator $S=-k_{\mathrm{B}} B \ln \rho$ (always well-defined). For independent states, $S=$ $S_{A} \otimes I_{B}+I_{A} \otimes S_{B}=-k_{\mathrm{B}}\left[B_{A} \ln \rho_{A} \otimes I_{B}+I_{A} \otimes B_{B} \ln \rho_{B}\right]$. For permanently non-interacting subsystems, every trajectory passing through a state in which the subsystems are in independent states must proceed through independent states along the entire trajectory, i.e., when two uncorrelated systems do not interact with each other, each must evolve in time independently of the other.

In addition, if at some instant of time two subsystems $A$ and $B$, not necessarily non-interacting, are in independent states, then the instantaneous rates of change of the subsystem's entropy functionals $-k_{\mathrm{B}} \operatorname{Tr}\left(\rho_{A} \ln \rho_{A}\right)$ and $-k_{\mathrm{B}} \operatorname{Tr}\left(\rho_{B} \ln \rho_{B}\right)$ must both be nondecreasing in time.

9. Correlations, entanglement and locality. Strong separability. Two non-interacting subsystems $A$ and $B$ initially in correlated and/or entangled states (possibly due to a previous interaction that has then been turned off) should in general proceed in time towards less correlated and entangled states. In any case, in order for the dynamics not to generate locality problems, i.e., faster-than-light communication between noninteracting subsystems (even if in entangled or correlated states), entanglement and correlations must not increase in the absence of interactions. In other words, when subsystem $A$ is not interacting with subsystem $B$, it should never be possible to influence the local observables of $A$ by acting only on the interactions within $B$, such as switching on and off parameters or measurement devices within $B$.

This however does not mean that existing entangle- ment and/or correlations between $A$ and $B$ established by past interactions should have no influence whatsoever on the time evolution of the local observables of either $A$ or $B$. In particular, there is no physical reason to request that two different states $\rho$ and $\rho^{\prime}$ such that $\rho_{A}^{\prime}=\rho_{A}$ should evolve with identical local dynamics $\left(\mathrm{d} \rho_{A}^{\prime} / \mathrm{d} t=\mathrm{d} \rho_{A} / \mathrm{d} t\right)$ whenever $A$ does not interact with $B$, even if entanglement and/or correlations in state $\rho$ differ from those in state $\rho^{\prime}$. Rather, the two local evolutions should be different until spontaneous decoherence (if any) will have fully erased memory of the entanglement and the correlations established by the past interactions now turned off. In fact, this may be a possible experimental scheme to detect spontaneous decoherence.

Compatibility with the predictions of QM about the generation of quantum entanglement between interacting subsystems that emerge through the Schrödingervon Neumann term $-i[H, \rho] / \hbar$ of Eq. (1), requires that the dissipative term $D_{M}$ may entail (spontaneous) loss of entanglement and loss of correlations between subsystems, but should not be able to create them.

10. Onsager reciprocity. First, we introduce a particularly useful representation of general nonequilibrium states [7]. Given any state $\rho$ on $\mathcal{H}$, we define the effective Hilbert space $\mathcal{H}^{\prime}$ as above, and choose a set of operators $\left\{I^{\prime}, X_{1}^{\prime}, X_{2}^{\prime}, \ldots\right\}$ spanning the linear space $\mathcal{L}_{h}\left(\mathcal{H}^{\prime}\right)$ of linear hermitian operators on $\mathcal{H}^{\prime}$; the corresponding state $\rho^{\prime}$ on $\mathcal{H}^{\prime}$ has no zero eigenvalues, so that $S=-k_{\mathrm{B}} B \ln \rho$ becomes $S^{\prime}=-k_{\mathrm{B}} \ln \rho^{\prime}$ on $\mathcal{H}^{\prime}$, which can be written as $S^{\prime}=f_{0} I^{\prime}+\sum_{j} f_{j} X_{j}^{\prime}$ because it belongs to $\mathcal{L}_{h}\left(\mathcal{H}^{\prime}\right)$. Thus,

$$
\rho^{\prime}=\frac{\exp \left(-\sum_{j} f_{j} X_{j}^{\prime} / k_{\mathrm{B}}\right)}{\operatorname{Tr} \exp \left(-\sum_{j} f_{j} X_{j}^{\prime} / k_{\mathrm{B}}\right)},
$$

where $f_{0}=k_{\mathrm{B}} \ln \operatorname{Tr} \exp \left(-\sum_{j} f_{j} X_{j}^{\prime} / k_{\mathrm{B}}\right)$. Similarly, we can also write $S_{e}^{\prime}=-k_{\mathrm{B}} \ln \rho_{e}^{\prime}=f_{0 e} I^{\prime}+\sum_{j} f_{j e} X_{j}^{\prime}$, for the target maximum-entropy equilibrium state on $\mathcal{H}^{\prime}$

$$
\rho_{e}^{\prime}\left(\rho^{\prime}\right)=\frac{\exp \left(-\beta H^{\prime}+\sum_{k} \nu_{k} G_{k}^{\prime}\right)}{\operatorname{Tr} \exp \left(-\beta H^{\prime}+\sum_{k} \nu_{k} G_{k}^{\prime}\right)},
$$

where $\beta$ and $\nu_{k}$ are such that $e\left(\rho_{e}^{\prime}\right)=e\left(\rho^{\prime}\right)$ and $g_{i}\left(\rho_{e}^{\prime}\right)=g_{i}\left(\rho^{\prime}\right)$, so that $\operatorname{Tr}\left(\rho^{\prime} \ln \rho_{e}^{\prime}\right)=\operatorname{Tr}\left(\rho_{e}^{\prime} \ln \rho_{e}^{\prime}\right)$ and $\operatorname{Tr}\left[\left(\mathrm{d} \rho^{\prime} / \mathrm{d} t\right) S_{e}^{\prime}\right]=0$. As a result, the following relations can be easily proved,

$$
\begin{gathered}
s\left(\rho^{\prime}\right)-s\left(\rho_{e}^{\prime}\left(\rho^{\prime}\right)\right)=f_{0}-f_{0 e}+\sum_{i}\left(f_{i}-f_{i e}\right) x_{i}\left(\rho^{\prime}\right), \\
\frac{\partial\left[s\left(\rho^{\prime}\right)-s\left(\rho_{e}^{\prime}\left(\rho^{\prime}\right)\right)\right]}{\partial x_{i}\left(\rho^{\prime}\right)}=f_{i}-f_{i e} \\
\frac{\mathrm{d} s\left(\rho^{\prime}\right)}{\mathrm{d} t}=\sum_{i} f_{i} \frac{\mathrm{D} x_{i}\left(\rho^{\prime}\right)}{\mathrm{D} t}=\sum_{i}\left(f_{i}-f_{i e}\right) \frac{\mathrm{D} x_{i}\left(\rho^{\prime}\right)}{\mathrm{D} t} \\
\left\langle\Delta S^{\prime} \Delta S^{\prime}\right\rangle=\sum_{i j} f_{i} f_{j}\left\langle\Delta X_{i}^{\prime} \Delta X_{j}^{\prime}\right\rangle
\end{gathered}
$$

where $\mathrm{D} x_{i}\left(\rho^{\prime}\right) / \mathrm{D} t=\operatorname{Tr}\left(D_{M} X_{i}^{\prime}\right)$ denotes the dissipative rate of change of the linear mean-value functional $x_{i}\left(\rho^{\prime}\right)=\operatorname{Tr}\left(\rho^{\prime} X_{i}^{\prime}\right),\left\langle\Delta S^{\prime} \Delta S^{\prime}\right\rangle=\operatorname{Tr}\left[\rho^{\prime}\left(-k_{\mathrm{B}} \ln \rho^{\prime}\right)^{2}\right]-$ 
$s\left(\rho^{\prime}\right)^{2},\left\langle\Delta X_{i}^{\prime} \Delta X_{j}^{\prime}\right\rangle=\frac{1}{2} \operatorname{Tr}\left[\left(\rho\left\{X_{i}^{\prime}, X_{j}^{\prime}\right\}\right]-x_{i}\left(\rho^{\prime}\right) x_{j}\left(\rho^{\prime}\right)\right.$. When the system is in state $\rho^{\prime}$, we interpret $\left\langle\Delta X_{i}^{\prime} \Delta X_{j}^{\prime}\right\rangle$ as the codispersion (covariance) of simultaneous measurements of observables $X_{i}^{\prime}$ and $X_{j}^{\prime},\left\langle\Delta X_{i}^{\prime} \Delta X_{i}^{\prime}\right\rangle$ as the dispersion (or fluctuations) of observable $X_{i}^{\prime}$ and $\left\langle\Delta S^{\prime} \Delta S^{\prime}\right\rangle$ the entropy fluctuations.

By Eq. (10), we may also interpret $f_{i}-f_{i e}$ as the generalized affinity or force conjugated with the mean value of the linear observable $X_{i}$. In order to recover Onsager's theory, we may impose that (at least in the vicinity of state $\rho_{e}^{\prime}$ ) the extended dynamics be such that the dissipative rates be linearly related to the generalized affinities through generalized-conductivity functionals, i.e.,

$$
\frac{\mathrm{D} x_{i}\left(\rho^{\prime}\right)}{\mathrm{D} t}=\sum_{j} L_{i j}\left(\rho^{\prime}, H^{\prime}, G_{k}^{\prime}, X_{\ell}^{\prime}, \ldots\right)\left(f_{j}-f_{j e}\right)
$$

where the $L_{i j}$ 's may be nonlinear functionals of $\rho^{\prime}$ (possibly to be approximated with their values at $\rho_{e}^{\prime}$, in its vicinity), but should form a symmetric $(\mathbf{H} \rightarrow-\mathbf{H}$, if $H^{\prime}$ depends on an external magnetic field) non-negative definite matrix, so that the rate of entropy production results in a quadratic form $\sum_{i j}\left(f_{i}-f_{i e}\right) L_{i j}\left(f_{j}-f_{j e}\right)$. Moreover, the $L_{i j}$ 's should be linearly interrelated with the matrix of codispersions $\left\langle\Delta X_{i}^{\prime} \Delta X_{j}^{\prime}\right\rangle$, in order to recover also Callen's fluctuation-dissipation theorem.

* Electronic address: beretta@unibs.it

[1] An account of this vaste literature can be found in the following papers and references therein: S. Weinberg, Phys. Rev. Lett. 62, 485 (1989); A. Stern, Y. Aharonov, and Y. Imry, Phys. Rev. A 41, 3436 (1990); A. K. Ekert, Phys. Rev. Lett. 67, 661 (1991); J. A. Holyst and L. A. Turski, Phys. Rev. A 45, 6180 (1992); G. Vidal and R. F. Werner, Phys. Rev. A 65, 032314 (1993); W. G. Unruh and R. M. Wald, Phys. Rev. D 52, 2176 (1995); C. H. Bennett et al., Phys. Rev. Lett. 76, 722 (1996); M. Grigorescu, Physica A 256, 149 (1998); A. Miranowicz, H. Matsueda and M. R. B. Wahiddin, J. Phys. A 33, 5159 (2000).

[2] See, e.g., G. J. Milburn, Phys. Rev. A 44, 5401 (1991); M. Hensel and H. J. Korsch, J. Phys. A 25, 2043 (1992); M. R. Gallis, Phys. Rev. A 48, 1028 (1993); A. K. Rajagopal, Phys. Rev. A 54, 1124 (1996); B. Reznik, Phys. Rev. Lett. 76, 1192 (1996); and references therein

[3] G. Domokos and S. Kovesi-Domokos, J. Phys. A 32, 4105 (1999).

[4] M. Czachor, Phys. Rev. A 57, 4122 (1998); M. Czachor and J. Naudts, Phys. Rev. E 59, R2497 (1999).

[5] S. Gheorghiu-Svirschevski, Phys. Rev. A 63, 022105 and 054102 (2001).

[6] See, e.g., V.I. Yukalov, Phys. Rev. Lett. 90, 167905 (2003) and references therein.

[7] G. P. Beretta, Found. Phys. 17, 365 (1987). See the references therein for credit to where a general explicit form of $\hat{\mathbf{D}}_{M}$ for steepest-entropy-ascent quantum dynamics was first formulated and where the conceptual need to remove the restriction $\rho^{2}=\rho$ was first advocated.
[8] R. Englman, Appendix in M. Lemanska and Z. Jaeger, Physica D 170, 72 (2002).

[9] D. Aerts et al., Phys. Rev. E 67, 051926 (2003).

[10] By strictly isolated we mean that the system interacts with no other systems and at some time (and, hence, at all times) is in an independent state when viewed as a subsystem of any conceivable composite system containing it.

[11] See references [1] in Ref. [5].

[12] Given a $\rho, B=B^{2}=I-P_{\operatorname{Ker}(\rho)}=P_{\perp \operatorname{Ker}(\rho)}$, so that $B=B^{2}, B \rho=\rho B=\rho$, and $B=B_{A} \otimes B_{B}$ when $\rho=$ $\rho_{A} \otimes \rho_{B}$. Moreover, from $B=B^{2}$ follows that $B \dot{B} B=$ 0 and $\operatorname{Tr}(\rho \dot{B} \ln \rho)=\operatorname{Tr}(B \dot{B} B \rho \ln \rho)=0$, thus $\dot{s}(\rho)=$ $\operatorname{Tr}(\dot{\rho} S)-k_{\mathrm{B}} \operatorname{Tr}(\dot{\rho})$ with $S=-k_{\mathrm{B}} B \ln \rho$.

[13] For a discussion on the relation between the notion of stability in thermodynamics and the mathematical concept of stability see Ref. [15]. The relevant definitions of local and global stability and of metastabilty are as follows.

An equilibrium state is stable in the sense required by the second law if it can be altered to a different state only by interactions that leave net effects in the state of the enviromment, i.e., alter the values of the energy and the other invariants. We call this notion global stability. Lyapunov local stability is necessary for global stability, but not sufficient: we must exclude metastability. As a result, the concept of global stability implied by the second law is as follows. An equilibrium state $\rho_{e}$ is globally stable if for every $\eta>0$ and every $\epsilon>0$ there is a $\delta(\epsilon, \eta)>0$ such that every trajectory $u(t, \rho)$ with $\eta<d\left(u(0, \rho), \rho_{e}\right)<\eta+\delta(\epsilon, \eta)$, i.e., passing at time $t=0$ between distance $\eta$ and $\eta+\delta$ from $\rho_{e}$, remains within $d\left(u(t, \rho), \rho_{e}\right)<\eta+\epsilon$ for every $t>0$, i.e., proceeds in time without ever exceeding the distance $\eta+\epsilon$.

The dynamical law may admit many equilibrium states that all share the same values of the invariants $\operatorname{Tr}(\rho H)$ and $\operatorname{Tr}\left(\rho G_{i}\right)$ and the parameters embedded in the Hilbert space $\mathcal{H}$ and the Hamiltonian $H$ describing the external forces (such as the size of a container), but among all these only one must globally stable, i.e., all the other equilibrium states must either be unstable or metastable.

[14] Under a unitary (Hamiltonian) dynamical law the trajectories would be $u(t, \rho)=U(t) \rho U^{-1}(t)$ with $U(t)=$ $\exp (-i t H / \hbar)$. The equilibrium states $\rho_{e}$, with $\rho_{e} H=$ $H \rho_{e}$, would all be globally stable. Indeed, with respect to the metric $d\left(\rho_{1}, \rho_{2}\right)=\operatorname{Tr}\left|\rho_{1}-\rho_{2}\right|$, it is easy to show that every trajectory $u(t, \rho)$ would be equidistant from any given equilibrium state $\rho_{e}$, i.e., $d\left(u(t, \rho), \rho_{e}\right)=$ $d\left(u(0, \rho), \rho_{e}\right)$ for all $t$ and all $\rho$. Because for each set of values of the invariant functianal these globally stable equilibrium states are in general more than one, the second-law requirement of uniqueness would be violated.

[15] G. P. Beretta, J. Math. Phys. 27, 305 (1986). The conjecture therein was later found proved in F. Hiai, M. Ohya, and M. Tsukada, Pacific J. Math. 96, 99 (1981).

[16] Some recent nonextensive quantum theories are based on other well-behaved entropy functionals such as the Daroczy-Tsallis functional. However [see e.g., E.P. Gyftopoulos and E. Çubukçu, Phys. Rev. E 55, 3851 (1997)], compatibility with thermodynamics requires the Gibbs-Shannon-von Neumann functional $s(\rho)=$ $-k_{\mathrm{B}} \operatorname{Tr}(\rho \ln \rho)$. 